\title{
Isolation of Circulating Tumor Cells and their Potential Heterogeneity Analysis in Advanced Non-Small Cell Lung Cancer
}

\author{
Qi Zhang ${ }^{1}$, JingYing Nong', Jinghui Wang ${ }^{1}$, Zhuohong Yan ${ }^{2}$, Ling $\mathrm{Yi}^{2}$, Xu Cheng ${ }^{3}$, Zongde Zhang ${ }^{4}$ Zhidong Liu $^{3}$, \\ Hongtao Zhang ${ }^{2}$ and Shucai Zhang*
}

${ }^{1}$ Department of Medical Oncology, Capital Medical University, China

${ }^{2}$ Department of Central Laboratory, Capital Medical University, China

${ }^{3}$ Department of Thoracic Surgery, Capital Medical University, China

${ }^{4}$ Department of Beijing Key Laboratory for Drug Resistant Tuberculosis Research, China

Submission: November 21, 2017; Published: November 05, 2017

"Correspondence Address: Shucai Zhang, Department of Medical Oncology, Beijing Chest Hospital, Capital Medical University, Beijing Tuberculosis and Thoracic Tumor Research Institute, No.97 Machang, Tongzhou Distric., Beijing 101149, China, E-mail: sczhang6304@163.com

Hongtao Zhang, Department of Central Laboratory, Beijing Chest Hospital, Capital Medical University and Beijing Tuberculosis and Thoracic Tumor Research Institute, No. 97 Machang, Tongzhou District, Beijing 101149, China, Email: zhtbeijing@163.com

\begin{abstract}
Background: Circulating tumor DNA offers a "real-time" tool for serial monitoring of tumor genomes by providing accessible genetic biomarkers for cancer diagnosis, prognosis, and response to therapy; thus, circulating tumor DNA has progressively become routinely used in clinical oncology. Another type of liquid biopsy focuses on analysis of circulating tumor cells (CTCs), which is potentially more practical for obtaining genome-wide information and facilitating heterogeneity analysis. The Cell Search System is the only validated method for CTC detection that has been approved by the Food and Drug Administration. Based on this principle, we enrolled a group of patients with newly diagnosed and locally advanced or metastatic non-small cell lung cancer, CTCs were isolated by micromanipulation followed by wholegenome amplification, then analyzed by quantitative PCR for epidermal growth factor receptor (EGFR) mutation. In $63 \%$ of these patients $\geq 1$ CTC was isolated. We then investigated whether the captured CTCs were suitable for molecular characterization. Four patients, the amplified CTC genomic DNA was further analyzed that proved with copy number variation. However, only two of these patients' CTCs showed exactly specific EGFR mutations identical to those of the primary tumor, while the others showed clearly different mutations from the primary tumor. Conclusions: These preliminary results suggest that the use of CTCs may be feasible for heterogeneity analysis of tumor mutations.
\end{abstract}

Keywords: Metastatic NSCLC; Circulating tumor cells; Heterogeneity

Abbreviations: NSCLC: Non-Small-Cell Lung Cancer; CTCs: Circulating Tumor Cells; EGFR: Epidermal Growth Factor Receptor Gene

\section{Introduction}

In China, lung cancer is the most commonly diagnosed cancer, and the leading cause of cancer death [1]. Therapeutic progress for non-small-cell lung cancer (NSCLC) subtypes is largely attributable to the accumulation of molecular knowledge and the development of corresponding drugs that specifically target molecular abnormalities. Patients whose neoplasms have specific genomic aberrations have great benefited from molecular targeted therapies [2]. Nearly 50\% of NSCLC found in Asian patients show mutations, especially in advanced lung adenocarcinoma [3]. Use of tyrosine kinase inhibitors (TKIs) to target EGFR-mu tated NSCLC is initially effective, but limited by the emergence of other mutations that confer drug resistance.

Resistance to TKI inhibitors, including first-generation and second-generation drugs (such as erlotinib, gefitinib and afatinib), usually occurs after a median period of 10-12 months of initially starting these agents. The T790M mutation is the most common cause of drug resistance; up to $60 \%$ of acquired resistance to TKIs in NSCLC treatment is reportedly due to this secondary mutation [4]. Although resistance to EGFR-TKIs is usually due to acquisition of secondary mutations in EGFR, whether 
T790M mutations pre-exist in clones prior to TKI treatment or evolve in response to treatment is not simple identified, both scenarios have clinical implications with respect to third-generation TKIs [5]. Molecular characterizations of biopsies from different regions within individual tumors or from primary tumors and their metastases have shown substantial heterogeneity [6]. A single sample might be insufficient to accurately represent all the resistance mechanisms. Tumor heterogeneity may be a key issue for EGFR-TKI resistance.

Liquid biopsy as a non-invasive testing method is becoming attractive, not only for evaluating prognosis, but also for detecting molecular drivers of tumors by which response or resistance to targeted agents could be predicted in terms of personalized treatment. As mentioned before, tumor biopsies that are limited to single sites may be unsuitable for characterizing a full tumor profile with respect to intermetastatic and intratumoral heterogeneity. Use of circulating tumor DNA (ctDNA) may be a practicable "real time" means of monitoring tumor genomes by providing accessible genetic biomarkers for cancer diagnosis, prognosis and response to therapy. Analyses of ctDNAs have been used to monitor response to treatment, and assess emergence of TKI resistance [7]. Another liquid biopsy technique focuses on analysis of circulating tumor cells (CTCs), and is potentially a more feasible means of obtaining genome-wide information and facilitating heterogeneity analysis. The Cell Search system is the only validated method for CTC detection that has been approved by FDA. Detection of CTCs in some patients with the use of magnetic bead-conjugated antibodies against epithelial cell adhesion molecule (EpCAM) is useful as a prognostic marker [8].

Based on the Cell Search method to isolate CTCs, we have enrolled 60 patients with newly diagnosed and locally advanced or metastatic NSCLC. From $63 \%$ of these patients, we have isolated $\geq 1$ CTC each (median: 2 CTCs, range: $0-18$ cells per $4.0 \mathrm{ml}$ blood). We then investigated whether the captured CTCs were suitable for molecular characterization. Four patients recruited whose primary tumor mutations had been tested and the amplified CTCs' genomic DNA was further analyzed to confirm copy number variations (CNV). In two out of four patients, CTCs (3and 5 CTCs, respectively) were detected with exactly the same mutation, whereas the other two had different mutations from the primary tumor.

\section{Conclusion}

Our preliminary results suggest that use of CTCs may be a feasible approach for heterogeneity analysis of tumor mutations.

\section{Acknowledgement}

This works supported by grants of "Study on the Application of Clinical Characteristics of Capital" ZHT (Z151100004015104) and ZSC (Z161100000516107) from Beijing Municipal Science and Technology Project.

\section{Conflict of Interest}

The authors declare no conflict of interest.

\section{Your next submission with Juniper Publishers will reach you the below assets}

- Quality Editorial service

- Swift Peer Review

- Reprints availability

- E-prints Service

- Manuscript Podcast for convenient understanding

- Global attainment for your research

- Manuscript accessibility in different formats

( Pdf, E-pub, Full Text, Audio)

- Unceasing customer service

Track the below URL for one-step submission https://juniperpublishers.com/online-submission.php 